\title{
Testing the Buffering Effect of Social Relationships in a Prospective Study of Disability Onset
}

\author{
Richard E. Lucas ${ }^{1} \&$ William Chopik ${ }^{1}$ \\ ${ }^{1}$ Department of Psychology, Michigan State University
}

\begin{abstract}
Author Note
This research supported by National Institute on Aging Grant AG040715 awarded to Richard E. Lucas. This document uses unit record data from the Household, Income and Labour Dynamics in Australia (HILDA) Survey. The unit record data from the HILDA Survey was obtained from the Australian Data Archive, which is hosted by The Australian National University. The HILDA Survey was initiated and is funded by the Australian Government Department of Social Services (DSS) and is managed by the Melbourne Institute of Applied Economic and Social Research (Melbourne Institute). The findings and views based on the data, however, are those of the authors and should not be attributed to the Australian Government, DSS, the Melbourne Institute, the Australian Data Archive or The Australian National University and none of those entities bear any responsibility for the analysis or interpretation of the unit record data from the HILDA Survey provided by the authors. Code to reproduce these analyses (along with additional supplemental analyses) is available at: https://osf.io/tjbxn/?view_only=0b71c9fb56474e57894043c16849ab6b
\end{abstract}




\section{Author Note}

Correspondence concerning this article should be addressed to Richard E. Lucas, 316

Physics Rd., Michigan State University, East Lansing, MI 48823. E-mail: lucasri@msu.edu 


\begin{abstract}
Social support has been proposed to be a protective factor that buffers the losses that result from the experience of negative life events. The present study uses data from a large-scale Australian panel study (the Household, Income and Labour Dynamics in Australia study) to examine how life satisfaction changes following the onset of a disabling condition and then to test whether pre-event or post-event social support moderates reactions to this event. Results show that the onset of a disabling condition is associated with a large decline in life satisfaction but these changes are not moderated by pre-event social support. Post-event social support does moderate change in response to the onset of a disability, but ambiguities in the interpretation of this association must be considered. Abstract Word Count: 129
\end{abstract}

Keywords: life satisfaction, disability, social relationships, subjective well-being, longitudinal

Word count: 4,944 (Text, Abstract, and Acknowledgements) 


\section{Testing the Buffering Effect of Social Relationships in a Prospective Study of Disability Onset}

Subjective well-being (SWB) reflects people's subjective evaluation of the quality of their lives as a whole (Diener et al., 2018). A critical question concerns which external circumstances affect individual differences in SWB. Laypeople might be interested in this question because answers could provide useful information about how to improve the quality of their lives. Social scientists are interested in this question because the answers inform core theoretical debates in their fields. Thus, identifying which life circumstances matter has been a central goal for many years.

Although early evidence suggested that life circumstances have only short-lived effects on SWB (e.g., Brickman et al., 1978), a closer look at this evidence revealed some reasons to question this broad conclusion. Most importantly, some of the most salient and widely cited studies suffered from a broad range of limitations including small sample sizes, questionable interpretations of results, and an over-reliance on cross-sectional designs for testing questions about change over time (Lucas, 2007). Researchers have since used longitudinal studies to assess whether changes in life circumstances are associated with changes in SWB. These studies show that life events and life circumstances do matter for SWB: Events and experiences, such as widowhood, eunemployment, and the onset of a disability, appear to be associated with lasting changes in SWB (Lucas et al., 2003, 2004; Lucas, 2007; Luhmann et al., 2012).

As evidence has accumulated that life circumstances matter, attention has turned toward identifying factors that moderate these associations. By determining who adapts best following major setbacks or who sustains lasting boosts following triumphs and achievements, it might be possible to identify successful strategies for improving people's lives. In addition, by studying which factors promote resilience in the face of major life changes, a better understanding of the process by which these circumstances affect SWB can be obtained. 
One promising potential moderator is social support. Many variables related to the quantity and quality of people's social relationships are associated with both of psychological and physical health (House et al., 1988; Myers, 1999; Uchino et al., 1996). Thus it seems reasonable that these factors may be especially important when life changes occur. Indeed, social support researchers have long suggested that social support can buffer the effects of various stressors (Cohen \& Wills, 1985).

Prospective longitudinal designs allow for especially strong tests of buffering effects (Lakey \& Cronin, 2008). This is because prospective studies assess moderators before life events occur. Researchers can rule out the possibility that interaction effects result from a causal effect of adaptation on the moderator. For instance, in a cross-sectional study, evidence for buffering might be that those who have high social support report greater well-being than those with less social support following the experience of a major life event. An alternative explanation of this effect, however, is that those who adapt best attract more social support or even just report more social support than those who adjust less well. By assessing the moderator before the event in a prospective design, this alternative explanation can be ruled out ${ }^{1}$.

In a test of these buffering effects, Anusic and Lucas (2014) used three prospective studies to assess whether pre-event social relationships buffered the effects of widowhood on life satisfaction. Although social relationships assessed after the loss of a spouse did moderate the association between the event and self-reported life satisfaction, pre-event relationship variables did not. One concern about Anusic et al's results, however, is that widowhood is intricately involved in social support (Ha, 2008). This means that the event in question may affect the moderating variables in ways that do not generalize to other events. In the current study we test whether a measure of social support moderates adaptation to

\footnotetext{
1 Though of course, it is still possible that some third variable causes both individual differences in the moderator and better adaptation, so causal effects of the moderator cannot be assumed.
} 
the onset of a serious disability.

\section{Disability and Social Support}

Examining social support as moderator of adaptation to the onset of a disability is a promising approach for at least two reasons. First, disability has consistently been shown to be associated with reduced levels of SWB (Bierman, 2012; Dijkers, 1997; Freedman et al., 2017) and there is considerable variability in reactions to disability (Lucas, 2007). Thus, this event provides an opportunity to investigate individual differences in changes that occur. Second, social support is thought to operate through a range of mechanisms, including the provision of social companionship and advice, along with more tangible help with household chores and financial assistance (Cohen \& Wills, 1985). Because the onset of a serious disability can lead to a broad range of life changes that would benefit from help from others (Bierman \& Statland, 2010), it is plausible that social support would have a buffering effect.

Multiple studies have examined the extent to which various forms of social support provide a buffer against the declines that are associated with disability. Variables such as marital status, marital support, and family support have been shown to moderate the association between impairment status and SWB (e.g., Carr et al., 2017, 2019; Bierman, 2012; Bookwala, 2011; Chan et al., 2011; Mancini \& Bonanno, 2006). Most studies that have examined these buffering effects, however, use cross-sectional designs or post-event longitudinal designs to assess interactions between social support and the existence or severity of health conditions or limitations.

In the current study, we use prospective, longitudinal data to test whether a measure of perceived social support ${ }^{2}$ moderates the association between disability onset and life

\footnotetext{
${ }^{2}$ Social support researchers often distinguish between "perceived support," which reflects respondents' perception and evaluation of the quality of their support and "enacted social support," which focuses more on the receipt of supportive behaviors. The former tend to be more strongly associated with health and well-being (Haber et al., 2007). In the current study, the available measure is more closely reflective of perceived support than enacted support.
} 
satisfaction. We first attempt to replicate the basic association between change in disability status and change in life satisfaction that has been found in German and British samples (Lucas, 2007). We then test whether pre-existing individual differences in social support and social relationships moderate the association with this life event. Evidence for a buffering effect would be found if those who started the study with more supportive social relationships suffered less of a decline after the onset of the disability.

\section{Methods}

\section{Participants}

Participants come from first 13 waves of the Household, Income, and Labour Dynamics in Australia survey (HILDA; Watson \& Wooden, 2012), a panel study that includes a nationally representative sample of households in Australia. Households were selected using a multi-stage approach, where Australian districts were randomly selected, and then households were randomly selected within districts. All household members age 15 and older were asked to participate. Surveys were administered in both face-to-face and self-report questionnaire format. Detailed information about survey characteristics and sampling methods can be found in the HILDA User Manual (Summerfield et al., 2014). Because only de-identified, existing data were accessed, this project is not considered human subjects research.

Participants were selected for inclusion if, at the start of the study, they did not have a disability that impacted their ability to work, acquired such a disability at some point in the study, and then remained in that disability status for the duration of their time in the study (see below for our operationalization of disability). Although additional respondents acquire and then recover from disabilities in this sample, by focusing only on those who acquire a disability and then maintain that disability status, we can assess long-term adaptation, which is our goal. Among all participants, 279 met inclusion criteria. The average age of these participants was $49.97(\mathrm{SD}=17.84)$ and $60.43 \%$ were women. 
Respondents participated for an average of 6.15 years before onset and 5.38 after.

Sample size was not determined in advance; rather all participants from the parent study who met inclusion criteria were included in the final sample. Power to detect the effect of disability found in prior studies is approximately $100 \%$. Indeed, our study has approximately $95 \%$ power to detect disability effects that are approximately one-third the size found in Lucas (2007). Power calculations for the moderating effect of social support are more difficult to calculate, as they require many assumptions. However, our simulations suggest that our study has approximately $90 \%$ power to detect an effect in which a one-standard deviation increase in social support reduces the effect of disability by $25 \%$. Detailed power analyses are available on the associated OSF page.

Our data access agreement prevents us from sharing the data, but interested researchers can obtain the data from survey organizers after signing a confidentiality agreement and paying a small fee. Our OSF page provides scripts that extract and clean the raw data and reproduce results reported in this manuscript. Because we have been working with these data for many years and have considerable knowledge of them, analyses were not preregistered.

\section{Measures}

The primary outcome measure is life satisfaction, which was measured each year with a single item that read "All things considered, how satisfied are you with your life? Again, pick a number between 0 and 10 to indicate how satisfied you are." Participants were responded on a scale ranging from 0 "Totally dissatisfied" to 5 "Neither satisfied nor dissatisfied" to 10 "Totally satisfied". Prior research suggests that single-item measures like this provide scores with reasonable reliability and validity in contexts like this (Cheung \& Lucas, 2014; Lucas \& Donnellan, 2012).

Disability status was assessed using two questions (both of which were presented each 
year). First, participants were shown a list of health conditions (full list available here: https://melbourneinstitute.unimelb.edu.au/assets/documents/hildaquestionnaires/ShowcardsW1.pdf) and asked "do you have any long-term health condition, impairment or disability (such as these) that restricts you in your everyday activities, and has lasted or is likely to last, for 6 months or more?" Those who indicated that they did have a health condition were then asked whether the conditions they listed "limit the type of work or the amount of work that you can do." Participants were classified as having a disability if they answered "yes" to both questions. To ensure that participants were moving from a state of relative health to one of lasting disability, only those with at least three years of data before and after onset were retained for analyses ${ }^{3}$.

Social support was assessed each year using the mean of 10 questions. These included questions about whether people had others they could confide in, whether they had a lot of friends, whether they can find support that they need, and whether they feel lonely (e.g., For exact wording, see the list provided at our OSF site). Participants responded on a 7-point scale ranging from 1 "strongly disagree" to 7 "strongly agree." Cronbach's alpha across all participants and all waves for this ten-item measure was .82. Separate pre-disability and post-disability social support scores were calculated by first averaging scores from all waves before onset (pre-disability social support) and from all waves after onset (post-disability social support) $)^{4}$.

${ }^{3}$ Relying only on the first question increases the sample size by 28 respondents and reduces the overall effect of disability slightly (as would be expected, as the conditions that are included are likely less severe). The pattern of associations with social support are the same (see our OSF page).

${ }^{4}$ Exploratory analyses revealed that the measures had a two-factor structure, with all positively worded items loading on one factor, and all negatively worded items loading on a second factor. Subscales based on these factors correlated moderately with one another, and the same pattern of results was found for each subscale as was found with the full measure (see OSF page). Given the issues with factor interpretability, we chose to present the results from the full measure here, but we report the disaggregated results on our OSF page. 


\section{Analytic Strategy}

A multilevel modeling strategy was used to assess within-person changes in life satisfaction following the onset of a disabling condition. Specifically, we used the lme4 (Bates et al., 2015) and lmerTest (Kuznetsova et al., 2017) packages in R to estimate multilevel models predicting yearly life satisfaction from disability status. In the simplest model, we predicted life satisfaction from a dichotomous variable indicating whether the respondent reported being disabled at the time of the survey. In this model, the intercept reflects average life satisfaction prior to the onset of the disabling condition and the coefficient for the disability predictor reflects difference in average life satisfaction from before to after onset. Existing research shows that people's life satisfaction can start to decline prior to the onset of disability. This may be because conditions that cause disability can emerge (and affect life satisfaction) before they become severe enough to affect one's ability to work (Lucas, 2007). Because of this, we also included a dummy variable that is coded 1 in the year before the onset of the disabling condition and 0 for all other waves.

In a second model, we add a linear change component to assess whether respondents adapt back towards baseline levels of life satisfaction. The linear change variable is coded 0 in all years up to and including the first year of disability and then increases by one for each additional year. Thus, after inclusion of this variable in the model, the coefficient for the dichotomous "disability" variable now reflects the change in life satisfaction from baseline to the first year of disability and the estimated linear change coefficient reflects the yearly change in life satisfaction following onset. In all models, the intercept, disability, and linear effects varied randomly.

To test the moderating effect of social support, we included average social support as a moderator of all within-person effects. Specifically, in one model, we used pre-disability social support as a moderator. In a second model, we replaced pre-disability social support with post-disability support. And finally, in a third model, we included both pre- and 
post-disability social support. As noted below, the linear change effect was not significant, so it was removed for the focal models, though analyses including this component can be found on our OSF page.

Full code to reproduce the analyses reported in this paper is included in the Rmarkdown document that is posted on our OSF page. We used R (Version 4.0.3; R Core Team, 2020) $)^{5}$ for all of our analyses.

\section{Results}

We first fit a null model that estimated an overall average life satisfaction score across all waves and participants, along with within- and between-person standard deviations. The overall mean was 7.71 and the within- and between-person standard deviations were 1.30 and 1.25 , respectively. The average pre-disability social support score was $5.29(\mathrm{SD}=0.83)$ and the average post-disability social support score was 5.17 ( $\mathrm{SD}=0.92)$. A paired-sample $t$-test showed that social support scores were significantly lower after onset of the disabling condition than before, $t(266)=3.59, p<.001$, though the effect size was small, reflecting a standardized mean difference of just 0.17 standard deviations (using the pre-disability standard deviation to standardize). Stability in social support from before to after onset of the disability was strong: $r=.75,95 \%$ CI $[.69, .80], t(265)=18.30, p<.001$. The average age at the time of disability was $56.38(\mathrm{SD}=18.02)$.

We next added the dichotomous disability and year-before-onset indicators to the model. As can be seen in Table 1, there is a small but significant drop from baseline in the year before respondents start reporting their disability, and a much larger drop after onset. This drop represents a decline of 0.60 standard deviations relative to average pre-disability

\footnotetext{
${ }^{5}$ We, furthermore, used the R-packages citr (Version 0.3.2; Aust, 2019), dplyr (Version 1.0.2; Wickham et al., 2020), ggplot2 (Version 3.3.2; Wickham, 2016), ggrepel (Version 0.8.2; Slowikowski, 2020), gridExtra (Version 2.3; Auguie, 2017), lme4 (Version 1.1.23; Bates et al., 2015), lmerTest (Version 3.1.2; Kuznetsova et al., 2017), papaja (Version 0.1.0.9942; Aust \& Barth, 2020), readr (Version 1.3.1; Wickham et al., 2018), tidyr (Version 1.1.2; Wickham \& Henry, 2020), and tidyverse (Version 1.3.0; Wickham et al., 2019).
} 


\section{Table 1}

Baseline Model Reporting Change in Life Satisfaction from Before to After Onset of Disabling Condition

\begin{tabular}{lrrrrrr}
\hline & Estimate & SE & CI & df & t & p \\
\hline Intercept & 8.06 & 0.08 & {$[7.91,8.22]$} & 293.43 & 101.39 & 0.00 \\
Year Before & -0.17 & 0.08 & {$[-0.32,-0.02]$} & $2,721.64$ & -2.15 & 0.03 \\
Disability & -0.73 & 0.07 & {$[-0.87,-0.58]$} & 298.79 & -9.80 & 0.00 \\
\hline
\end{tabular}

Note. $\mathrm{N}=279 . \mathrm{CI}=95 \%$ confidence interval.

life satisfaction. Thus, using standard rules of thumb for evaluating effect sizes, the decline is relatively large and consistent with prior work (Lucas, 2007).

We also tested whether age or gender moderated any of these associations. There was only a significant moderating effect of gender on change in life satisfaction in the year before onset, with men reporting a larger decline during that year than women. Because age and gender were not associated with the focal effects of this or any subsequent analyses, and because we had no a priori hypotheses about such moderating effects, these variables were not included in the models reported below (details are included in the supplemental analyses on our OSF page).

We next assessed whether respondents returned to baseline following onset by adding a linear change parameter. The coefficient for the linear change component was not significant: Estimate $=-0.04, S E=0.02,95 \% C I=[-0.08,0], d f=157.53, t=-1.93, p=$ 0.06. In fact, the small negative coefficient is in the opposite direction to what would be expected if respondents adapted back to baseline. This is consistent with previous studies (Lucas, 2007).

To assess whether there is evidence that social support buffers reactions to the onset of a disability, we added grand-mean-centered pre-event social support as a person-level 
Table 2

Model Reporting Moderating Effect of Pre-Disability Social Support on Change in Life Satisfaction from Before to After Onset of Disabling Condition

\begin{tabular}{lrrrrrr}
\hline & Estimate & SE & CI & df & \multicolumn{1}{c}{$\mathrm{t}$} & $\mathrm{p}$ \\
\hline Intercept & 8.05 & 0.07 & {$[7.92,8.19]$} & 295.77 & 116.05 & 0.00 \\
Year Before & -0.16 & 0.08 & {$[-0.32,-0.01]$} & $2,723.84$ & -2.06 & 0.04 \\
Disability & -0.72 & 0.07 & {$[-0.86,-0.57]$} & 296.50 & -9.61 & 0.00 \\
Pre-Disability Social Support & 0.79 & 0.08 & {$[0.62,0.96]$} & 300.51 & 9.35 & 0.00 \\
Year-Before X Social Support & 0.04 & 0.10 & {$[-0.14,0.23]$} & $2,739.07$ & 0.46 & 0.64 \\
Disability X Social Support & 0.01 & 0.09 & {$[-0.17,0.18]$} & 301.80 & 0.08 & 0.94 \\
\hline
\end{tabular}

Note. $\mathrm{N}=278 . \mathrm{CI}=95 \%$ confidence intervals

predictor of the within-person changes that occurred after onset. Results are presented in Table 2. Social support was positively associated with the intercept: Those who reported higher social support before onset also reported high levels of life satisfaction during that same period. The critical question, however, is whether the social resources that were amassed when things were going well (i.e., social support in the pre-disability period) buffered the loss in life satisfaction that occurred following onset. The last two lines of Table 2 show that there is no support for this idea: Social support was not associated with the changes that occurred before and after disability onset.

The left panel of Figure 1 plots predicted trajectories for those at the mean level of social support, one standard deviation above the mean, and one standard deviation below the mean. As can be seen in the figure (and as reflected in Table 2), the predicted trajectories are almost perfectly parallel. Those who have high levels of social support before the onset of the disabling condition report higher levels of life satisfaction, but social support does not protect against declines. Those with high social support decline as much as those with low social support did. Again, this demonstrates that there is no buffering effect of pre-disability social support in this sample. The differences in life satisfaction between those high and low in pre-disability social support reflect the differences that existed before the 


\section{Table 3}

Model Reporting Moderating Effect of Post-Disability Social Support on Change in Life Satisfaction from Before to After Onset of Disabling Condition

\begin{tabular}{lrrrrrr}
\hline & Estimate & SE & CI & df & t & $\mathrm{p}$ \\
\hline Intercept & 8.08 & 0.07 & {$[7.93,8.22]$} & 283.48 & 109.21 & 0.00 \\
Year Before & -0.16 & 0.08 & {$[-0.31,0]$} & $2,638.48$ & -1.97 & 0.05 \\
Disability & -0.74 & 0.07 & {$[-0.88,-0.59]$} & 286.58 & -10.00 & 0.00 \\
Post-Disability Social Support & 0.53 & 0.08 & {$[0.37,0.68]$} & 283.83 & 6.54 & 0.00 \\
Year-Before X Social Support & 0.29 & 0.09 & {$[0.12,0.47]$} & $2,648.20$ & 3.33 & 0.00 \\
Disability X Social Support & 0.42 & 0.08 & {$[0.27,0.58]$} & 286.68 & 5.30 & 0.00 \\
\hline
\end{tabular}

Note. $\mathrm{N}=269 . \mathrm{CI}=95 \%$ confidence intervals.

onset of the disabling condition.

Next we tested the moderating association of post-disability social support on each of the within-person parameters from the baseline model. Results are shown in Table 3. In contrast to the results for pre-disability social support, many estimated coefficients from this model are significantly different from zero, including the moderating associations with social support. The right panel of Figure 1 plots the implied trajectories from this model. As can be seen in this figure, respondents who reported high levels of social support (i.e., those who scored one standard deviation above the mean) after the onset of a disability exhibited declines in life satisfaction that were less than half the size of those who reported low levels of post-disability social support. Again, however, it is important to caution that the interpretation of this post-disability effect is more complicated than that for pre-disability social support. Post-disability social support may either serve a buffering function or it may actually be caused by better adaptation: Those who fare best after the onset of a disability may be more likely to attract (or report) higher social support.

Finally, although there was strong stability in social support from before to after onset of the disabling condition, it is possible to examine the associations between social 

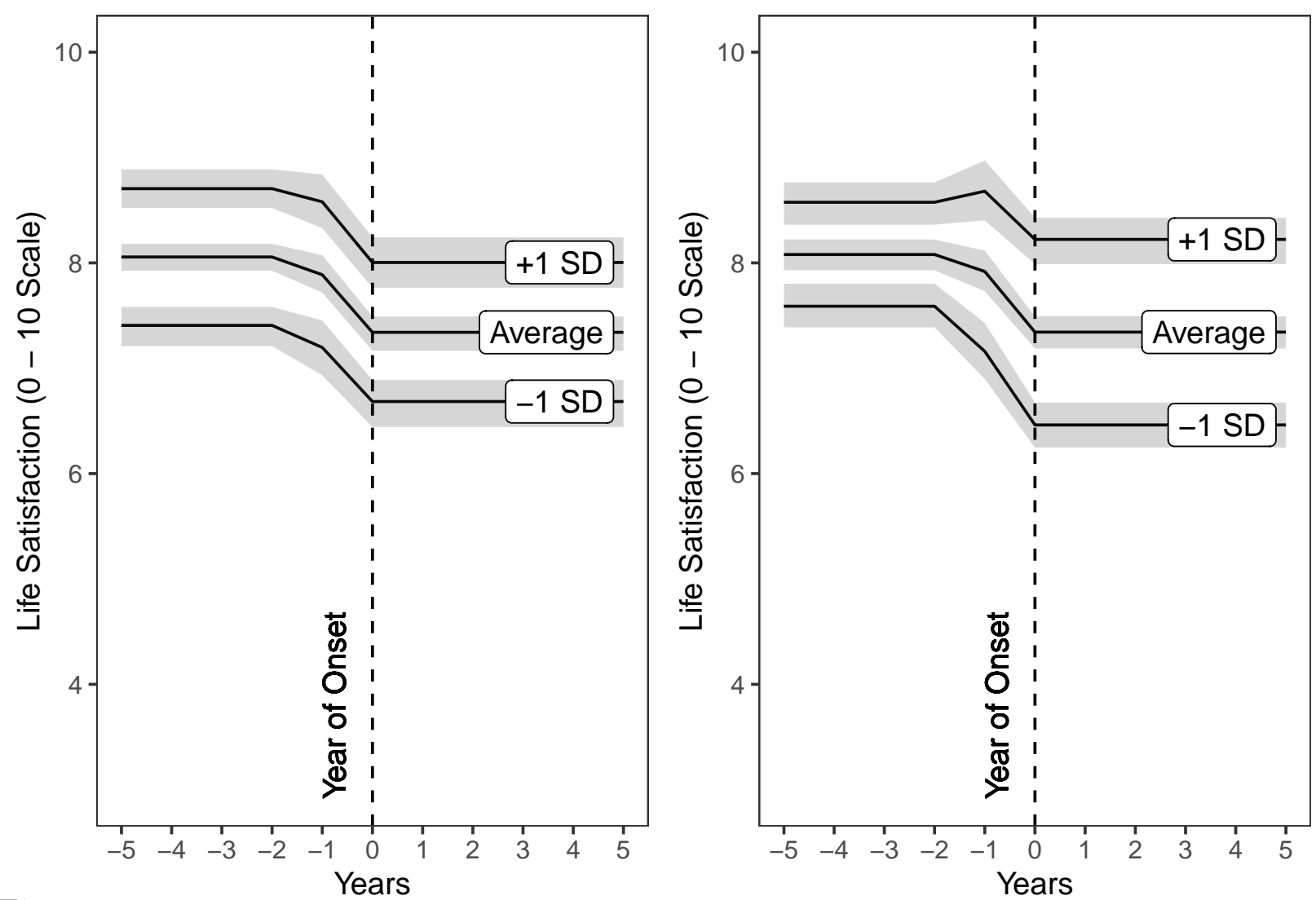

Figure 1

Pre-Disability and Post-Disability Social Support as Moderators of Adaptation to Disability. Figure by Lucas (2020); available at https://doi.org/10.6084/m9.figshare.12403964.v2, under a $C C$-BY license.

support in one period and changes in life satisfaction, while holding social support from the other period constant. Table 4 shows the results of an analysis that includes both pre- and post-disability social support as moderators. Holding post-disability social support constant, pre-disability social support is positively associated with pre-disability life satisfaction and negatively associated with the changes that occur in the year before onset and in the years after. In other words, having high social support before disability onset does not appear to be beneficial if that especially high level of support is not maintained once the disability emerges. In contrast, post-disability social support is unrelated to pre-disability life satisfaction (controlling for pre-disability social support), but is associated with the changes 
Table 4

Model Reporting Moderating Effect of Pre-and Post-Disability Social Support on Change in Life Satisfaction from Before to After Onset of Disabling Condition

\begin{tabular}{lrcrrrr}
\hline & Estimate & SE & CI & df & t & $\mathrm{p}$ \\
\hline Intercept & 8.05 & 0.07 & {$[7.91,8.19]$} & 284.27 & 114.18 & 0.00 \\
Year Before & -0.14 & 0.08 & {$[-0.29,0.02]$} & $2,639.25$ & -1.71 & 0.09 \\
Disability & -0.71 & 0.07 & {$[-0.84,-0.57]$} & 283.07 & -10.12 & 0.00 \\
Pre-Disability Social Support & 0.78 & 0.13 & {$[0.53,1.03]$} & 292.02 & 6.00 & 0.00 \\
Post-Disability Social Support & 0.01 & 0.12 & {$[-0.22,0.23]$} & 286.15 & 0.08 & 0.94 \\
Year Before X Pre-Disability SS & -0.42 & 0.15 & {$[-0.71,-0.13]$} & $2,648.42$ & -2.82 & 0.00 \\
Disability X Pre-Disability SS & -0.77 & 0.13 & {$[-1.02,-0.52]$} & 288.49 & -5.98 & 0.00 \\
Year Before X Post-Disability SS & 0.57 & 0.13 & {$[0.31,0.83]$} & $2,643.21$ & 4.29 & 0.00 \\
Disability X Post-Disability SS & 0.93 & 0.11 & {$[0.71,1.15]$} & 282.44 & 8.19 & 0.00 \\
\hline
\end{tabular}

Note. $\mathrm{N}=268$. CI $=95 \%$ confidence interval.

in life satisfaction that occur following disability.

Figure 2 plots the predicted trajectories with the moderating effect of pre- and post-disability social support, each controlling for the other. The left panel shows the associations with pre-disability social support and the right panel shows the associations with post-disability social support. Again, these figures demonstrate that those with high levels of pre-disability social support are predicted to have no benefit from social support if it is not maintained in the post-disability period (i.e., if during this latter period, they report average levels of social support). This figure clarifies that although pre-disability social support is associated with pre-disability life satisfaction, it is the social support from the post-disability period that is most important for predicting adaptation to the onset of a disabling condition.

\section{Discussion}

A critical goal for SWB research is to identify the extent to which life events and changes in life circumstances are associated with changes in SWB. The first contribution of 

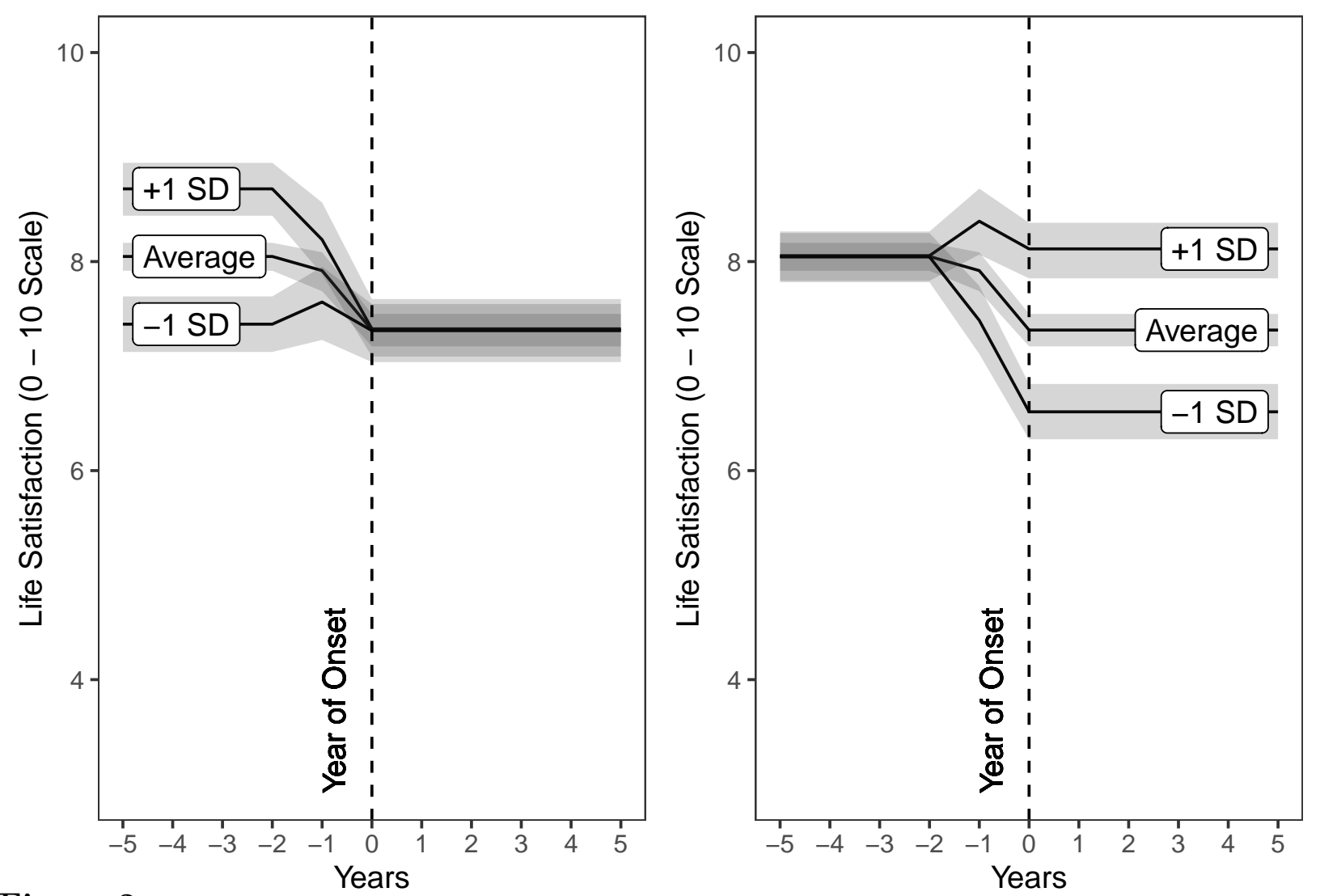

Figure 2

Pre-Disability and Post-Disability Social Support as Simultaneous Moderators of Adaptation to Disability. Figure by Lucas (2020); available at https://doi.org/10.6084/m9 figshare. 12403964.v2, under a CC-BY license.

the current paper was to replicate in a new, nationally representative sample, the finding that the onset of a disability is associated with changes in life satisfaction (Lucas, 2007). People who experienced this life event declined in their levels of life satisfaction, the change was large (approximately three-quarters of a standard deviation), and life satisfaction did not, on average, return to baseline levels.

The second major goal of this study was to use a prospective design to determine whether the social support that one obtains when things are going well can protect against declines in SWB when misfortune happens. Our use of a prospective design has one critical advantage over previous research that looks at buffering effects of social support: In studies 
that rely either on cross-sectional designs or designs that focus on social support that is reported after the onset of a negative event, any association between social support and improved functioning could either be due to the buffering effects of social support or to the fact that those who function best are able to attract or report greater social support.

Although the use of a prospective design cannot clearly establish the causal impact of social support, it can at least rule out the possibility that reactions to the event are causing the reported levels of social support. In these data, pre-existing social support did not predict better adaptation to the onset of a disability when tested using these prospective methods. This is consistent with prior prospective research that has often failed to find protective effects of social support when predicting the onset of depression (e.g., Lakey \& Cronin, 2008), and our study extends these findings to buffering effects in the context of a specific major life event.

To be clear, this study does not rule out a causal effect of social support on adaptation to disability. Indeed, our analyses showed that social support reported during the period of disability was associated with a reduced impact of the disabling event. This finding is consistent with previous research showing that various forms of social support interact with disability status to predict concurrent levels of well-being and related outcomes (e.g., Bierman, 2012; Bierman \& Statland, 2010; Bookwala, 2011; Carr et al., 2019, 2017; Mancini \& Bonanno, 2006; Schulz \& Decker, 1985). One possible interpretation of the combined findings is that it is only the social support that people are able to obtain and maintain during a negative period that predicts how one will function during that period; support built up prior to that time may not matter for post-disability adjustment. Our results are also consistent with a simple "main-effect" model of the effects of social support (Cohen \& Wills, 1985). Again, however, we caution that a plausible alternative interpretation of this effect is simply that those who reacted most positively to the event were also able to obtain or perhaps simply perceive more social support in their lives (Lakey \& Cronin, 2008). 


\section{Limitations}

Although the use of a prospective design from a large panel study is a major strength of this work, the study is not without limitations. First, one disadvantage of using existing data is that investigators are beholden to the choices made by the survey organizers regarding the measures used to assess critical concepts. Debates exist in the literature about the relative merits of assessing "perceived support," which reflects' respondents' evaluation of the quality of support they receive, versus "enacted support," which focuses more on concrete support behaviors that others provide. The measure included in the HILDA appears to focus more on perceived support, which tends to be more strongly correlated with health and well-being outcomes than are measures of perceived support (Haber et al., 2007). Yet it is possible that other components or measures of social support might have different associations with these outcomes.

Relatedly, it is important to recognize that all measures in this study were self-report. The reliance on self-report measures of social support can lead to problems in the interpretation of buffering effects if halo biases or other shared method variance inflate correlations between constructs of interest. Although our prospective design can help rule out some of these concerns (e.g., we can rule out the possibility that changes that result from the onset of a disability cause pre-disability reports of social support), it cannot rule them all out. Thus, research that incorporates non-self-report measures of the primary constructs can help clarify these effects further ${ }^{6}$.

\section{Summary}

This study used a prospective design to assess whether pre-existing levels of social support moderated people's reactions to the onset of a disabling condition. Our study

\footnotetext{
${ }^{6}$ In supplemental analyses on our OSF site, we report results controlling for self-report measures of plausible third variables: Extraversion and neuroticism. These analyses do not change the pattern of results for social support.
} 
replicated prior results that disability is associated with large, lasting declines in life satisfaction, with little evidence of a return to previous levels. An important new contribution of this work was to show that levels of social support assessed before the onset of the disability did not predict greater adjustment to the condition: Those with high levels of social support dropped just as far in their life satisfaction as those with low social support following the onset of this condition. We did find that those who reported high levels of social support after the onset of the disabling condition reported smaller declines over time, but the interpretation of this effect is ambiguous: High levels of self-reported social support might simply result from better adaptation and adjustment. Thus, these results show the promise of using prospective designs to clarify when and how social support is related to SWB following major life events. 


\section{References}

Anusic, I., \& Lucas, R. E. (2014). Do Social Relationships Buffer the Effects of Widowhood? A Prospective Study of Adaptation to the Loss of a Spouse. Journal of Personality, 82(5), 367-378. https://doi.org/10.1111/jopy.12067

Auguie, B. (2017). GridExtra: Miscellaneous functions for "grid" graphics. https://CRAN.R-project.org/package=gridExtra

Aust, F. (2019). Citr: 'RStudio' add-in to insert markdown citations. https://CRAN.R-project.org/package $=$ citr

Aust, F., \& Barth, M. (2020). papaja: Create APA manuscripts with $R$ Markdown. https://github.com/crsh/papaja

Bates, D., Mächler, M., Bolker, B., \& Walker, S. (2015). Fitting linear mixed-effects models using lme4. Journal of Statistical Software, 67(1), 1-48. https://doi.org/10.18637/jss.v067.i01

Bierman, A. (2012). Functional Limitations and Psychological Distress: Marital Status as Moderator. Society and Mental Health, 2(1), 35-52. https://doi.org/10.1177/2156869312442884

Bierman, A., \& Statland, D. (2010). Timing, Social Support, and the Effects of Physical Limitations on Psychological Distress in Late Life. The Journals of Gerontology: Series B, 65B(5), 631-639. https://doi.org/10.1093/geronb/gbp128

Bookwala, J. (2011). Marital Quality as a Moderator of the Effects of Poor Vision on Quality of Life Among Older Adults. The Journals of Gerontology: Series B, 66B(5), 605-616. https://doi.org/10.1093/geronb/gbr091

Brickman, P., Coates, D., \& Janoff-Bulman, R. (1978). Lottery winners and accident victims: Is happiness relative?. Journal of Personality and Social Psychology, 
$36(8), 917-927$.

Carr, D., Cornman, J. C., \& Freedman, V. A. (2019). Do Family Relationships Buffer the Impact of Disability on Older Adults' Daily Mood? An Exploration of Gender and Marital Status Differences. Journal of Marriage and Family, 81(3), 729-746. https://doi.org/10.1111/jomf.12557

Carr, D., Cornman, J. C., \& Freedman, V. A. (2017). Disability and Activity-related Emotion in Later Life: Are Effects Buffered by Intimate Relationship Support and Strain? Journal of Health and Social Behavior, 58(3), 387-403.

https://doi.org/10.1177/0022146517713551

Chan, N., Anstey, K. J., Windsor, T. D., \& Luszcz, M. A. (2011). Disability and Depressive Symptoms in Later Life: The Stress-Buffering Role of Informal and Formal Support. Gerontology; Basel, 57(2), 180-189.

https://doi.org/10.1159/000314158

Cheung, F., \& Lucas, R. E. (2014). Assessing the validity of single-item life satisfaction measures: Results from three large samples. Quality of Life Research, 23(10), 2809-2818.

Cohen, S., \& Wills, T. A. (1985). Stress, Social Support, and the Buffering Hypothesis. Psychological Bulletin; Washington, Etc., 98(2), 310. https://doi.org/10.1037/0033-2909.98.2.310

Diener, E., Lucas, R. E., \& Oishi, S. (2018). Advances and Open Questions in the Science of Subjective Well-Being. Collabra: Psychology, 4(1). https://doi.org/10.1525/collabra.115

Dijkers, M. (1997). Quality of life after spinal cord injury: A meta analysis of the effects of disablement components. Spinal Cord, 35(12), 829-840. 
Freedman, V. A., Carr, D., Cornman, J. C., \& Lucas, R. E. (2017). Aging, mobility impairments and subjective wellbeing. Disability and Health Journal, 10(4), 525-531. https://doi.org/10.1016/j.dhjo.2017.03.011

Ha, J.-H. (2008). Changes in Support From Confidants, Children, and Friends Following Widowhood. Journal of Marriage and Family, 70(2), 306-318. https://doi.org/10.1111/j.1741-3737.2008.00483.x

Haber, M. G., Cohen, J. L., Lucas, T., \& Baltes, B. B. (2007). The relationship between self-reported received and perceived social support: A meta-analytic review. American Journal of Community Psychology, 39(1), 133-144. https://doi.org/10.1007/s10464-007-9100-9

House, J. S., Landis, K. R., \& Umberson, D. (1988). Social relationships and health. Science, 241(4865), 540-545. https://doi.org/10.1126/science.3399889

Kuznetsova, A., Brockhoff, P. B., \& Christensen, R. H. B. (2017). lmerTest package: Tests in linear mixed effects models. Journal of Statistical Software, 82(13), 1-26. https://doi.org/10.18637/jss.v082.i13

Lakey, B., \& Cronin, A. (2008). Low Social Support and Major Depression: Research, Theory and Methodological Issues. In Risk Factors in Depression (pp. 385-408). Elsevier.

Lucas, R. (2020). Social Support as a Moderator of Reaction and Adaptation to Disability Onset. https://doi.org/10.6084/m9.figshare.12403964.v1

Lucas, R. E. (2007). Long-term disability is associated with lasting changes in subjective well-being: Evidence from two nationally representative longitudinal studies. Journal of Personality and Social Psychology, 92(4), 717-730. https://doi.org/10.1037/0022-3514.92.4.717 
Lucas, R. E., Clark, A. E., Georgellis, Y., \& Diener, E. (2003). Reexamining adaptation and the set point model of happiness: Reactions to changes in marital status. Journal of Personality and Social Psychology, 84(3), 527-539.

Lucas, R. E., Clark, A. E., Georgellis, Y., \& Diener, E. (2004). Unemployment Alters the Set Point for Life Satisfaction. Psychological Science, 15(1), 8-13. https://doi.org/10.1111/j.0963-7214.2004.01501002.x

Lucas, R. E., \& Donnellan, M. B. (2012). Estimating the Reliability of Single-Item Life Satisfaction Measures: Results from Four National Panel Studies. Social Indicators Research, 3, 323-331.

Luhmann, M., Hofmann, W., Eid, M., \& Lucas, R. E. (2012). Subjective well-being and adaptation to life events: A meta-analysis. Journal of Personality and Social Psychology, 102(3), 592-615.

Mancini, A. D., \& Bonanno, G. A. (2006). Marital closeness, functional disability, and adjustment in late life. Psychology and Aging, 21(3), 600-610. https://doi.org/10.1037/0882-7974.21.3.600

Myers, D. G. (1999). Close relationships and quality of life. In D. Kahneman, E. Diener, \& N. Schwarz (Eds.), Well-being: The foundations of hedonic psychology (pp. 374-391). Russell Sage Foundation.

R Core Team. (2020). R: A language and environment for statistical computing. $\mathrm{R}$ Foundation for Statistical Computing. https://www.R-project.org/

Schulz, R., \& Decker, S. (1985). Long-term adjustment to physical disability: The role of social support, perceived control, and self-blame. Journal of Personality and Social Psychology, 48(5), 1162-1172. https://doi.org/10.1037/0022-3514.48.5.1162 
Slowikowski, K. (2020). Ggrepel: Automatically position non-overlapping text labels with 'ggplot2'. https://CRAN.R-project.org/package=ggrepel

Summerfield, M., Freidin, S., Hahn, M., Li, N., Macalalad, N., Mundy, L., Watson, N., Wilkins, R., \& Wooden, M. (2014). HILDA User Manual-Release 13.

Uchino, B. N., Cacioppo, J. T., \& Kiecolt-Glaser, J. K. (1996). The relationship between social support and physiological processes: A review with emphasis on underlying mechanisms and implications for health. Psychological Bulletin, 119 (3), 488-531. https://doi.org/10.1037/0033-2909.119.3.488

Watson, N., \& Wooden, M. (2012). The HILDA Survey: A Case Study in the Design and Development of a Successful Household Panel Study. Longitudinal and Life Course Studies, 3(3), 369-381.

Wickham, H. (2016). Ggplot2: Elegant graphics for data analysis. Springer-Verlag New York. https://ggplot2.tidyverse.org

Wickham, H., Averick, M., Bryan, J., Chang, W., McGowan, L. D., François, R., Grolemund, G., Hayes, A., Henry, L., Hester, J., Kuhn, M., Pedersen, T. L., Miller, E., Bache, S. M., Müller, K., Ooms, J., Robinson, D., Seidel, D. P., Spinu, V., ... Yutani, H. (2019). Welcome to the tidyverse. Journal of Open Source Software, 4 (43), 1686. https://doi.org/10.21105/joss.01686

Wickham, H., François, R., Henry, L., \& Müller, K. (2020). Dplyr: A grammar of data manipulation. https://CRAN.R-project.org/package=dplyr

Wickham, H., \& Henry, L. (2020). Tidyr: Tidy messy data. https://CRAN.R-project.org/package=tidyr

Wickham, H., Hester, J., \& Francois, R. (2018). Readr: Read rectangular text data. https://CRAN.R-project.org/package=readr 